\title{
Variabilidade espacial de parâmetros físico-químicas do solo e biofísicos de superfície em cultivo do sorgo
}

\author{
Alberto C. de C. Bernardi ${ }^{1}$, Ladislau M. Rabello², Ricardo Y. Inamasu ${ }^{3}$, \\ Célia R. Grego ${ }^{4}$ \& Ricardo G. Andrade 5 \\ ${ }^{1}$ Embrapa Pecuária Sudeste. São Carlos, SP. E-mail: alberto.bernardi@embrapa.br (Autor correspondente) \\ ${ }^{2}$ Embrapa Instrumentação. São Carlos, SP. E-mail: ladislau.rabello@embrapa.br \\ ${ }^{3}$ Embrapa Instrumentação. São Carlos, SP. E-mail: ricardo.inamasu@embrapa.br \\ ${ }^{4}$ Embrapa Monitoramento por Satélite. Campinas, SP. E-mail: celia.grego@embrapa.br \\ ${ }^{5}$ Embrapa Monitoramento por Satélite. Campinas, SP. E-mail: ricardo.andrade@embrapa.br
}

Palavras-chave:

condutividade elétrica do solo

Landsat 5 - TM

SEBAL

\begin{abstract}
R E S U M O
O conhecimento da variabilidade espacial das propriedades do solo e das culturas é importante para as tomadas de decisão sobre o manejo agrícola. Objetivou-se, neste trabalho, avaliar a variabilidade espacial de parâmetros químicos e físicos do solo e biofísicos de superfície de área cultivada com sorgo. O estudo foi conduzido em área de 12 ha de um Argissolo Vermelho Amarelo distrófico. A amostragem de solo georreferenciada e a medição da condutividade elétrica do solo foram realizadas antes do plantio do sorgo. As imagens do satélite Landsat 5 foram utilizadas para calcular os parâmetros biofísicos de superfície. Ferramentas de geoestatística foram utilizadas para se determinar e modelar a variabilidade espacial dos atributos em estudo em que os resultados mostraram que a densidade de amostragem adotada foi insuficiente para uma caracterização adequada da variabilidade espacial de parâmetros do solo. Ocorreu dependência espacial de grau, moderada a forte para CE e parâmetros biofísicos da superfície da imagem de satélite com alcances variando de 74,4 a 181,1 m; por outro lado, o sensoriamento remoto orbital também foi útil para o mapeamento da variabilidade espacial da cultura do sorgo e tem grande potencial para aplicação da Agricultura de Precisão.
\end{abstract}

Key words:

soil electrical conductivity

Landsat 5 - TM

SEBAL

\section{Spatial variability of physico-chemical parameters of soil and biophysical surface of sorghum crop}

\begin{abstract}
A B S T R A C T
The knowledge of spatial variability of soil and crop properties is useful for agricultural management decision making. The objective of this research was to evaluate the spatial variability of soil chemical and physical parameters and biophysical surface parameters of sorghum crop. The study was conducted in an area of 12 ha of a Typic Paleudult. Georrefered soil sampling and electrical conductivity (EC) of soil were conducted before sowing of sorghum. Landsat 5 imageries were used to establish the biophysical surface parameters. Geostatistics tool were used to determine and model the spatial variability of the attributes under study. Sampling density adopted was insufficient for an adequate characterization of the spatial variability of soil parameters. There was spatial dependence from moderate to strong degree for EC biophysical parameters with ranges varying from 74.4 to $181.1 \mathrm{~m}$. Remote sensing was useful to show spatial variability of sorghum crop and has a great potential for the application of precision agriculture.
\end{abstract}

\section{INTRODUÇÃO}

O estudo da variabilidade espacial em sistema de cultivo é de extrema importância para que se possa aprimorar as opções de manejo e melhorar a rentabilidade do sistema de produção. Para tanto, as ferramentas de agricultura de precisão (AP) devem ser implementadas partindo-se da premissa de que a técnica auxilia os produtores na tomada de decisões gerenciais para os diferentes sistemas de cultivo (Bramley, 2009). A AP requer ferramentas, como os sensores e as imagens de satélite, que possibilitem avaliar a variabilidade espacial do solo e a redução das amostragens intensivas e caras (Brevik et al., 2006).
A condutividade elétrica (CE) aparente do solo medida por sensores de contato ou por indução eletromagnética cujas leituras integram a textura e a disponibilidade de água, duas características do solo que afetam a produtividade e pode auxiliar na interpretação das variações de rendimento relacionadas com a variabilidade espacial da produção das culturas (Johnson et al., 2005). No Brasil, Machado et al. (2006) verificaram que os valores da CE estavam relacionados com o teor de argila do solo e sua variabilidade espacial e foram úteis para o estabelecimento dos limites de zonas de manejo em lavoura de soja. Em adição, os dados de sensoriamento remoto e as ferramentas de geoprocessamento têm sido fundamentais, por exemplo, para 
avaliação espacialmente explícita de parâmetros biofísicos de superfície (Numata et al., 2007; Andrade et al., 2012).

Uma das vantagens do sensoriamento remoto é a possibilidade de extração de informações das culturas em áreas extensas com rapidez e de forma menos onerosa, ou seja, com o mínimo de dados coletados no campo. Neste contexto os índices de vegetação obtidos por satélite podem ser utilizados para avaliar a biomassa de culturas e pastagens, tanto no espaço quanto no tempo.

O índice de vegetação da diferença normalizada (NDVI), por ser um índice de fácil aplicação e que possibilita rápida e eficiente detecção de variações na vegetação (Rouse et al., 1973) comumente é usado para avaliar a sanidade, a área de plantio, a biomassa e o teor de nutrientes das plantas (Freeman et al., 2007; Hatfield et al., 2008, Vicente et al., 2012). Alguns outros índices podem ser calculados a partir dessas faixas espectrais obtidas com sensores ativos, como índice de área foliar, teor de clorofila, biomassa, densidade, temperatura foliar e umidade (Hatfield et al., 2008).

O NDVI é um dos parâmetros biofísicos que vêm sendo utilizados como dado de entrada em modelos e algoritmos para obtenção de informações de superfície com poucos dados observacionais (Andrade et al., 2012), como o SEBAL (Surface Energy Balance Algorith for Land). O SEBAL possibilita, por exemplo, a estimativa dos componentes do balanço de energia e permite modificar e acoplar outros modelos em sua estrutura (Allen et al., 2002) facilitando as aplicações em estudos realizados tanto em escala local quanto na regional.

Neste trabalho avaliou-se a variabilidade espacial de parâmetros químicos e físicos do solo e biofísicos de superfície de área cultivada com sorgo.

\section{Material e Métodos}

O estudo foi conduzido em área de 12 ha na Embrapa

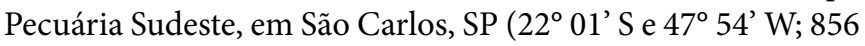
$m$ acima do nível do mar), em área de solo Argissolo VermelhoAmarelo distrófico textura média/argilosa. O clima da região é tropical de altitude, com histórico de $1502 \mathrm{~mm}$ de precipitação pluvial anual e médias de temperaturas mínima e máxima de $16,3^{\circ} \mathrm{C}$ (julho) e de $23^{\circ} \mathrm{C}$ (fevereiro), respectivamente.

A semeadura do sorgo (Sorghum bicolor $\mathrm{L}$. Moench) var. BRS 610 (híbrido) foi realizada em 14/11/2008 de forma a resultar em uma população de 5 plantas por metro linear e espaçamento entre linhas de $0,8 \mathrm{~m}$.

A amostragem de solo georreferenciada, realizada em $02 / 10 / 2008$, precedeu a semeadura do sorgo e foi feita utilizando-se uma grade de amostragem hexagonal com 6 subamostras para compor uma amostra composta sendo que cada hexágono cobria a área de 1 ha e as subamostras foram retiradas aleatoriamente, dentro dos limites do polígono. Foram coletadas 12 amostras e mais 8 pontos extras na transversal da área totalizando, assim, 20 amostras. A coleta de amostras de solo foi realizada com um quadriciclo equipado com GPS (Garmin GPS III Plus, com erro absoluto de posicionamento de $0,45 \mathrm{~m}$ no modo de operação com correção diferencial em tempo real) e trado de rosca de aço inoxidável com regulagem de profundidade e de acionamento elétricos, o que possibilitou a delimitação dos pontos com suas respectivas coordenadas geográficas.

As amostras de solo coletadas na profundidade de 0 a $0,2 \mathrm{~m}$, foram secadas ao ar, peneiradas em peneira de malha de $2 \mathrm{~mm}$ de acordo com Primavesi et al. (2005) e analisadas para determinação do $\mathrm{pH}$ em água, da matéria orgânica, do $\mathrm{P}$ disponível pelo método da resina, dos teores trocáveis de $\mathrm{K}$, $\mathrm{Ca}$ e $\mathrm{Mg}$, da acidez trocável e potencial e dos teores de argila e areia. Indiretamente foram calculadas, por meio do uso dos parâmetros anteriores, a soma de bases, a saturação por bases e a capacidade de troca de cátions (CTC) ao pH 7,0.

A condutividade elétrica (CE) do solo foi medida com o equipamento de fabricação da Veris Technologies, Salina, KS, modelo V3100. Esta medida ocorreu antes do plantio do sorgo nos dias 18 e 19/09/2008, fazendo-se um percurso por toda a área em estudo e se obtendo um conjunto de dados com 16.018 observações. Para estimativa do albedo, temperatura da superfície, NDVI, índice de área foliar, saldo de radiação e evapotranspiração real diária, foram utilizados o algoritmo SEBAL, as imagens do sensor TM do satélite Landsat 5 na data de 01/02/2009 (fase de florescimento do sorgo) e os dados meteorológicos da estação automática do Instituto Nacional de Meteorologia (INMET) localizada no município de São Carlos, SP, distante aproximadamente $3,5 \mathrm{~km}$ da área de estudo. De início, foram realizadas as devidas correções nas imagens e em seguida calculou-se a refletância monocromática de cada banda obtendo-se o NDVI e o SAVI (Soil Adjusted Vegetation Index).

No caso do NDVI o cálculo foi feito pela diferença de refletâncias entre a banda do infravermelho próximo (IVP) e a do vermelho (VERM), normalizada pela divisão da soma das bandas de IVP e VERM (Rouse et al., 1973) expressado pela Eq. 1.

$$
\mathrm{NDVI}=\frac{\rho_{\mathrm{IVP}}-\rho_{\mathrm{VERM}}}{\rho_{\mathrm{IVP}}+\rho_{\mathrm{VERM}}}
$$

em que:

$\rho_{\text {IVP }}$ e $\rho_{\text {VERM }}$ - refletâncias das bandas do vermelho e infravermelho próximo

OSAVI é um índice que reduz os efeitos do solo de fundo do NDVI para que impactos da umidade do solo sejam reduzidos no índice. O SAVI foi determinado de acordo com Huete (1988) por meio da Eq. 2.

$$
\mathrm{SAVI}=\frac{(1+\mathrm{L}) \times\left(\rho_{\mathrm{IVP}}-\rho_{\mathrm{VERM}}\right)}{\left(\mathrm{L}+\rho_{\mathrm{IVP}}+\rho_{\mathrm{VERM}}\right)}
$$

em que:

L - constante que varia entre 0 e 1 . Nesse estudo utilizouse $L$ igual a 0,5 (valor frequentemente encontrado na literatura).

De posse do SAVI estimou-se o índice de área foliar (IAF, dado em $\mathrm{m}^{2}$ folhas $\mathrm{m}^{-2}$ solo) por meio da equação empírica (Eq. 3) sugerida por Allen et al. (2002). 


$$
\mathrm{IAF}=-\frac{\ln \left(\frac{0,69-\mathrm{SAVI}}{0,59}\right)}{0,91}
$$

A próxima etapa foi a estimativa do albedo da superfície (Allen et al., 2002), expresso pela Eq. 4.

$$
\alpha=\frac{\alpha_{\text {toa }}-\alpha_{\mathrm{p}}}{\tau_{\mathrm{sw}}^{2}}
$$

em que:

a - albedo planetário

$\alpha_{\mathrm{p}} \quad$ - refletância atmosférica que varia entre 0,025 e 0,04 (utilizou-se o valor 0,03 )

$\tau_{\mathrm{sw}} \quad$ - transmissividade atmosférica

De posse do albedo da superfície deu-se início à aplicação de várias etapas descritas em detalhes por Allen et al. (2002) para estimar o fluxo de calor latente como um resíduo dos demais componentes da equação clássica do balaço de energia, conforme Eq. 5.

$$
\mathrm{LE}=\mathrm{Rn}-\mathrm{H}-\mathrm{G}
$$

em que:

$$
\begin{array}{ll}
\mathrm{LE} & \text { - fluxo de calor latente } \\
\mathrm{Rn} & \text { - saldo de radiação } \\
\mathrm{H} & \text { - fluxo de calor sensível } \\
\mathrm{G} & \text { - fluxo de calor no solo, todos em } \mathrm{W} \mathrm{m}^{-2}
\end{array}
$$

A partir dos componentes do balanço de energia foi possível calcular, por meio da Eq. 6 .

$$
\lambda=\frac{\mathrm{LE}}{\mathrm{LE}+\mathrm{H}}=\frac{\mathrm{LE}}{\mathrm{Rn}-\mathrm{G}}
$$

em que:

$\lambda$ - fração evaporativa, definida como a fração de calor latente para o máximo saldo de energia disponível
Estudos micrometeorológicos indicam que a fração evaporativa $(\lambda)$ pode ser considerada constante durante o período diurno; assim, para estimativa da evapotranspiração real diária $\left(\mathrm{ETr}_{\text {dérío }}, \mathrm{mm} \mathrm{d}^{-1}\right)$, em escala regional, foi empregada a Eq. (7) (Ayenew, 2003).

$$
\mathrm{ET}_{\text {diário }}=\frac{\lambda \mathrm{Rn}_{\text {diário }}}{28}
$$

em que:

$\mathrm{Rn}_{\text {diário }}$ - saldo de radiação obtido pela integração de dados meteorológicos no período de $24 \mathrm{~h}$

O escalar no denominador da Eq. 7 representa o fator de conversão de unidade $\left(\mathrm{W} \mathrm{m}^{-2}\right.$ para $\left.\mathrm{mm} \mathrm{d}^{-1}\right)$.

A partir dos dados (NDVI, albedo, Ts, IAF, $\left.\mathrm{Rn}_{\text {diário }} \mathrm{ETr}_{\text {diário }}\right)$ no formato "raster", no software ArcGis 9.2 (Environmental System Research Institute, Redland, CA), efetuou-se uma transformação para o formato "pontos/shape", obtendo-se 137 pontos. De posse dos dados de propriedades químicas e físicas do solo e biofísicos de superfície foi realizada a geoestatística; para tanto foi avaliada a existência de variabilidade espacial por meio da construção e ajuste de semivariogramas para representar a população do fenômeno em estudo através da função matemática utilizando o GEOEST (Vieira et al., 2002). Os dados de CE e parâmetros biofísicos de superfície, que apresentaram dependência espacial, foram espacializados pelo método de krigagem ordinária; já os dados das propriedades químicas do solo foram interpolados pelo método do inverso do quadrado da distância; posteriormente, utilizou-se o software Arc Gis 9.2 para gerar os mapas de contorno

\section{Resultados e Discussão}

\begin{tabular}{|c|c|c|c|c|c|c|c|c|}
\hline Variável & Média & Desvio padrão & Mínimo & Máximo & $n$ & CV (\%) & Curtose & Assimetria \\
\hline pH (água) & 6,33 & 0,18 & 6,00 & 6,70 & 20 & 2,76 & $-0,12$ & 0,14 \\
\hline $\mathrm{MO}\left(\mathrm{g} \mathrm{kg}^{-1}\right)$ & 43,60 & 4,87 & 35,00 & 55,00 & 20 & 11,17 & 0,19 & 0,49 \\
\hline $\mathrm{P}\left(\mathrm{mg} \mathrm{dm}^{-3}\right)$ & 36,35 & 20,52 & 9,00 & 87,00 & 20 & 56,45 & 0,61 & 0,93 \\
\hline $\mathrm{K}\left(\mathrm{cmol}_{\mathrm{c}} \mathrm{dm}^{-3}\right)$ & 1,42 & 0,34 & 0,80 & 2,20 & 20 & 23,86 & 0,69 & 0,49 \\
\hline CTC $\left(\mathrm{cmol}_{\mathrm{c}} \mathrm{dm}^{-3}\right)$ & 65,37 & 9,17 & 52,30 & 82,50 & 20 & 14,02 & $-0,84$ & 0,41 \\
\hline $\mathrm{V}(\%)$ & 66,68 & 6,66 & 54,80 & 79,40 & 20 & 10,00 & $-0,31$ & 0,12 \\
\hline Areia $\left(\mathrm{g} \mathrm{kg}^{-1}\right)$ & 653,35 & 19,23 & 627,00 & 691,00 & 20 & 2,94 & $-1,07$ & 0,15 \\
\hline Argila $\left(\mathrm{g} \mathrm{kg}^{-1}\right)$ & 284,30 & 38,64 & 215,00 & 351,00 & 20 & 13,59 & $-0,74$ & 0,056 \\
\hline $\mathrm{CE}\left(\mathrm{mS} \mathrm{m}^{-1}\right)$ & 2,94 & 1,43 & 0,40 & 11,10 & 16018 & 48,76 & 1,89 & $-0,03$ \\
\hline NDVI & 0,75 & 0,03 & 0,56 & 0,80 & 137 & 4,34 & 1,80 & 0,001 \\
\hline Albedo & 0,19 & 0,01 & 0,14 & 0,22 & 137 & 6,63 & 2,82 & $-1,13$ \\
\hline Ts & 17,26 & 1,83 & 15,52 & 23,89 & 137 & 10,58 & 2,35 & 1,66 \\
\hline IAF & 4,04 & 0,61 & 2,68 & 5,87 & 137 & 14,98 & 0,10 & 0,44 \\
\hline Rn diário & 120,76 & 4,29 & 108,55 & 137,78 & 137 & 3,55 & 2,92 & 0,71 \\
\hline ETr diário & 3,42 & 0,25 & 1,84 & 3,94 & 137 & 7,23 & 27,40 & $-4,57$ \\
\hline
\end{tabular}

Os dados estatísticos dos parâmetros de solo $\mathrm{pH}$ em água; matéria orgânica; $\mathrm{P}$ disponível pelo método da resina; teores de K trocável, areia e argila; saturação por bases, capacidade de troca de cátions (CTC) ao $\mathrm{pH} 7,0$, condutividade elétrica (CE) e do NDVI, são apresentados na Tabela 1.

Tabela 1. Estatísticas descritivas para atributos químicos e granulométricos do solo $(\mathrm{pH}, \mathrm{MO}, \mathrm{P}, \mathrm{K}, \mathrm{CTC}$, V\%, areia e argila), condutividade elétrica do solo (CE) e atributos biofísicos de superfície da imagem de satélite (NDVI, Albedo, Ts, IAF, Rn diário, ETr diário) de uma área cultivada com sorgo 
De acordo com Grego \& Vieira (2005) com base em trabalhos publicados anteriormente os valores de assimetria e curtose entre 0 e 3 indicam a distribuição normal dos dados; apenas os valores de $\mathrm{pH}, \mathrm{CTC}, \mathrm{V} \%$, areia e argila apresentam valores de assimetria e curtose não compatíveis com a normalidade (Tabela 1); os demais parâmetros apresentaram distribuição normal de acordo com o mesmo critério. Esta determinação é importante uma vez que a krigagem apresenta melhores resultados quando a normalidade dos dados é satisfeita; no entanto, a normalidade dos dados não é impedimento para o uso das técnicas de geoestatística.

$\mathrm{O}$ pH, saturação por bases (V\%), teor de areia e o NDVI, foram os parâmetros que apresentaram as menores magnitudes de variação, ou seja, coeficiente de variação $(\mathrm{CV})$ inferior a $10 \%$, considerado baixo (<10\%) por Gomes \& Garcia (1984). Os CV dos parâmetros matéria orgânica, argila e CTC, apresentaram $\mathrm{CV}$ médio entre 10 e $20 \%$, e o teor de $\mathrm{K}^{+}$apresentou $\mathrm{CV}$ de $24 \%$. Os valores do CV para $\mathrm{P}, \mathrm{Ke}$ CE foram considerados altos (Gomes \& Garcia, 1984).

De acordo com Kravchenko (2003) o nível de variabilidade dos dados é importante no manejo de sítios específicos visto que parâmetros do solo com alta variabilidade (como P e CE, no presente estudo) são potencialmente mais apropriados para serem manejados espacialmente que aqueles uniformemente distribuídos (como V\%). Por outro lado, o mapeamento de parâmetros do solo com alta variabilidade espacial pode ter menor acurácia que os valores com baixa variabilidade. As tendências de variação dos valores dos parâmetros de solo obtidos neste estudo estão de acordo com as observadas por Machado et al. (2004) e Kilic et al. (2012) para diversos parâmetros de solo.

Os parâmetros do solo (pH, MO, P, K, CTC, V\%, argila e areia) apresentaram efeito pepita puro indicando fraca dependência espacial (Vieira, 2000). É provável que tal ocorrência seja devida à baixa densidade de pontos da grade de amostragem utilizada (20 pontos). Como a variabilidade desses atributos é inferior à do espaçamento utilizado, seria necessário aumentar o número de pontos utilizados neste estudo.

Os semivariogramas experimentais para CE, albedo, temperatura da superfície (Ts), NDVI, índice de área foliar (IAF), saldo de radiação diário $\left(\mathrm{Rn}_{\text {dírio }}\right)$ e evapotranspiração real diária $\left(\mathrm{ETr}_{\text {dírio }}\right)$ foram calculados e os modelos ajustados foram delimitados (Tabela 2). O modelo esférico foi o que melhor se ajustou aos variogramas experimentais. Trangmar et al. (1985) já havia demonstrado que este modelo é o que melhor se ajusta para descrever o comportamento de semivariogramas de parâmetros do solo. Os alcances da dependência espacial indicados pelos modelos dos semivariogramas foram maiores para $\mathrm{ETr}_{\text {diário }}$ NDVI, Ts, IAF, $\mathrm{Rn}_{\text {diário }}$ e albedo, com valores de 181 a 94 . Mencionados resultados indicam que, para a área de estudo, grades de amostragem com espaçamentos de $90 \mathrm{~m}$ seriam adequadas para a caracterização da variabilidade espacial dos parâmetros biofísicos de superfície caso em que as imagens com resolução de $30 \times 30 \mathrm{~m}$ do sensor TM do satélite Landsat 5 são adequadas para este propósito; o mesmo ocorreu para as medidas de CE nas quais espaçamentos de $74 \mathrm{~m}$ poderiam ser utilizados para caracterizar sua variabilidade espacial. Os parâmetros biofísicos que apresentaram dependência espacial (Tabela 2), de acordo com os critérios de Cambardella et al. (1994) consideradas forte (efeito pepita $\leq 25 \%$ do patamar) foram para Ts, IAF, $\mathrm{Rn}_{\text {dírio }}$ e albedo, como dependência moderada (efeito pepita entre $26-75 \%$ do patamar), para CE, NDVI e ETr diário

Na Figura 1 visualiza-se a espacialização dos parâmetros de fertilidade do solo $\mathrm{pH}, \mathrm{P}, \mathrm{K}$, areia, argila, V\% e CTC da camada de 0-0,2 $\mathrm{m}$ de profundidade obtidos pelo inverso do quadrado da distância. Uma vez que não houve dependência espacial, os dados de solo (fertilidade e textura) não foram interpolados por krigagem optando-se por utilizar o interpolador inverso do quadrado da distância para elaboração dos mapas de contorno.

A acidez (Figura 1A) pode ser considerada muito baixa uma vez que os valores de $\mathrm{pH}$ são maiores que 6,0. Seguindo o critério de classificação de Raij et al. (1996), os valores para P (Figura 1B) foram considerados de baixo a médio (de $7 \mathrm{a} 40 \mathrm{mg} \mathrm{dm}^{-3}$ ) nas áreas claras do mapa, e altos (entre 41 e $80 \mathrm{mg} \mathrm{dm}^{-3}$ ) nas áreas mais escuras. Os valores de $\mathrm{K}$ (Figura $1 \mathrm{C}$ ) estiveram entre baixo $\left(0,8\right.$ a $\left.1,5 \mathrm{mmol}_{c} \mathrm{dm}^{-3}\right)$ e médio $\left(1,6\right.$ a $\left.3,0 \mathrm{mmol}_{c} \mathrm{dm}^{-3}\right)$ e a saturação por bases (Figura 1D) entre média (51 a 70\%) e alta (71 a 90\%). Tais valores indicam, na prática, que há diferenças dentro da área estudada e que essas diferenças poderiam ser corrigidas com o uso de aplicação de fertilizantes (com fósforo e potássio) em taxas variadas.

Como a medida de CE integra algumas propriedades do solo, como textura, matéria orgânica e CTC (Brevik et al., 2006) previa-se uma tendência desses mapas apresentarem o mesmo padrão; no entanto, o mapa de CE apresenta o mesmo padrão

Tabela 2. Parâmetros de ajuste do semivariograma para CE, albedo, temperatura da superfície (Ts), NDVI, índice de área foliar (IAF), saldo de radiação diário $\left(\mathrm{Rn}_{\text {diário }}\right)$ e evapotranspiração real diária $\left(\mathrm{ETr}_{\text {diário }}\right)$ de uma área cultivada com sorgo

\begin{tabular}{|c|c|c|c|c|c|}
\hline Parâmetros & $\begin{array}{c}\text { CO } \\
\text { (efeito pepita) }\end{array}$ & $\begin{array}{c}\text { C1 } \\
\text { (variância estrutural) }\end{array}$ & $\begin{array}{c}a \\
\text { (alcance) }\end{array}$ & $\begin{array}{l}\text { Modelo } \\
\text { de ajuste }\end{array}$ & $\begin{array}{c}\text { Dependência } \\
100\left[C_{0}\left(C_{0}+C_{1}\right)^{-1}\right]\end{array}$ \\
\hline CE & 0,9 & 0,448 & 74,39 & Esférico & 66,8 \\
\hline Albedo* & 0 & 0,00014252 & 94,19 & Esférico & 0,0 \\
\hline $\mathrm{Ts}^{*}$ & 0 & 1,8654 & 158,114 & Esférico & 0,0 \\
\hline $\mathrm{NDVl}^{*}$ & 0,000616 & 0,000386 & 161,72 & Esférico & 61,5 \\
\hline $\mathrm{IAF}^{*}$ & 0 & 0,31001 & 119,033 & Esférico & 0,0 \\
\hline $\mathrm{Rn}_{\text {diario }}{ }^{*}$ & 0 & 15,909 & 107,3 & Esférico & 0,0 \\
\hline$E \operatorname{Tr}_{\text {diaririo }}{ }^{*}$ & 0,018729 & 0,031098 & 181,069 & Esférico & 37,6 \\
\hline
\end{tabular}

* Fase de florescimento do sorgo (01/02/2009) 

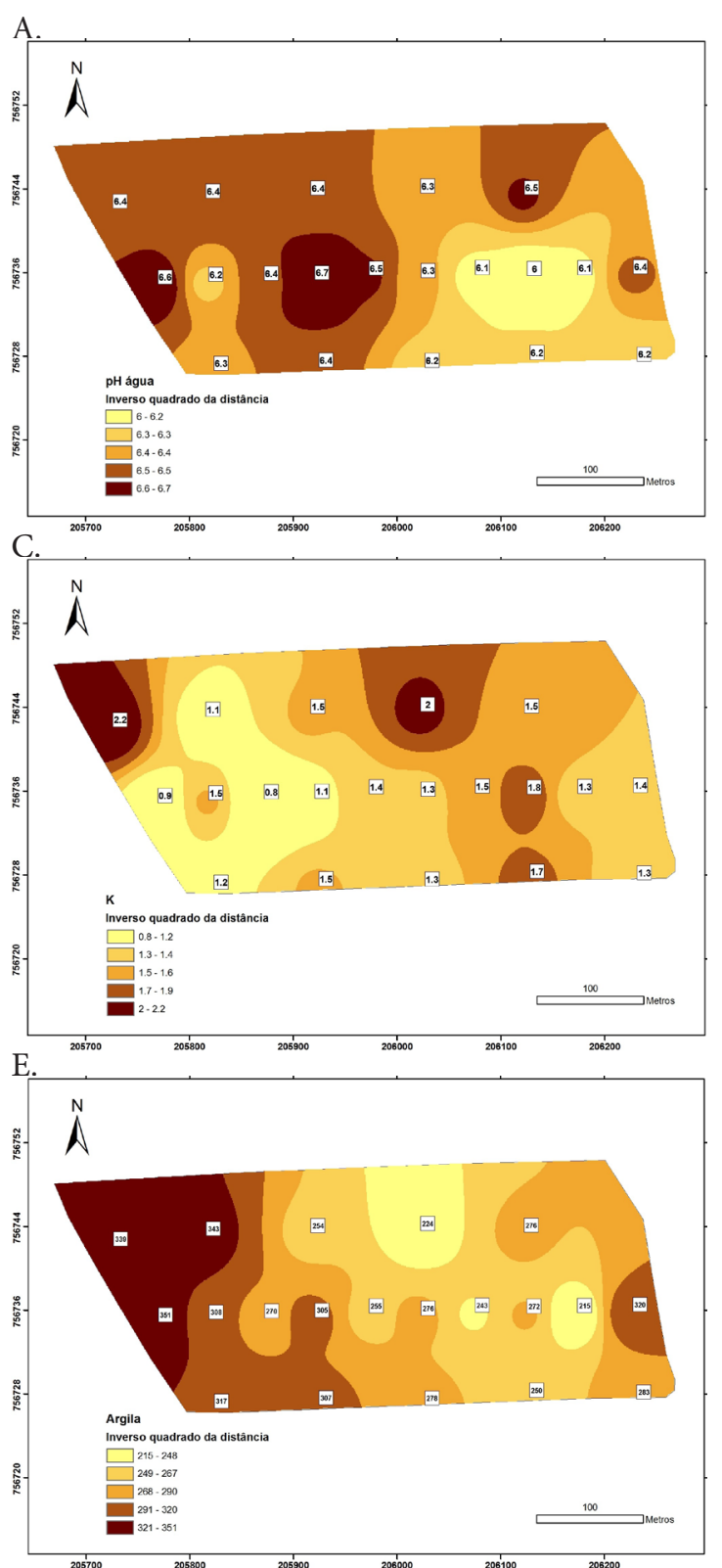
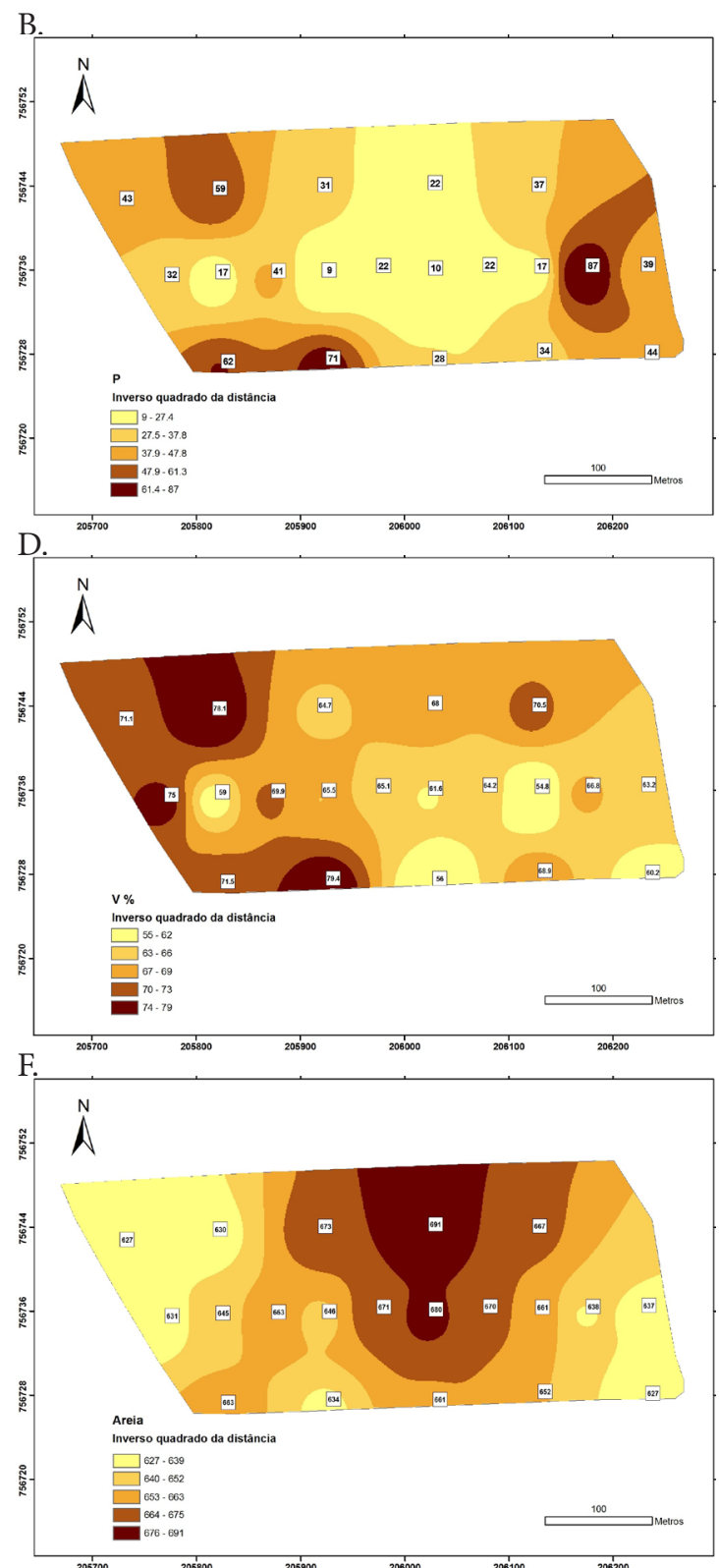

Figura 1. Mapas espacializados dos parâmetros de fertilidade do solo: $\mathrm{pH}(\mathrm{A})$, fósforo (B), potássio (C) (E) e areia (F) da camada de 0-20 cm de profundidade de uma área cultivada com sorgo

do teor de argila (Figuras 1E e 2) e já tinha observado, também, por Machado et al. (2006).

A Figura 3 apresenta, respectivamente, albedo, temperatura da superfície (Ts), NDVI, índice de área foliar (IAF), saldo de radiação diário $\left(\mathrm{Rn}_{\text {diário }}\right)$ e evapotranspiração real diária $\left(\mathrm{ETr}_{\text {diário }}\right)$ para a cultura do sorgo. Conforme a Figura $3 \mathrm{~A}, \mathrm{o}$ albedo variou de 0,141 a 0,220 , com média de 0,193 ; o valor mínimo de 0,141 teve ocorrência na região de borda e pode ter sido influenciado por vegetação da área externa (por exemplo, floresta); na maior parte da área de sorgo o albedo variou de 0,175 a 0,218. Mota et al. (1991) apresentaram valores de albedo para a cultura do sorgo variando de 0,18 a 0,23 ; com relação à temperatura da superfície, verificou-se oscilação entre 15,5 e $23,9^{\circ} \mathrm{C}$, sendo o valor médio de $17,3{ }^{\circ} \mathrm{C}$.

A região mais quente e a mais fria na área de sorgo se localizaram nas partes esquerda e direita da Figura 3B, respectivamente e, sem dúvida, isto ocorreu em razão do efeito de borda, além de que se

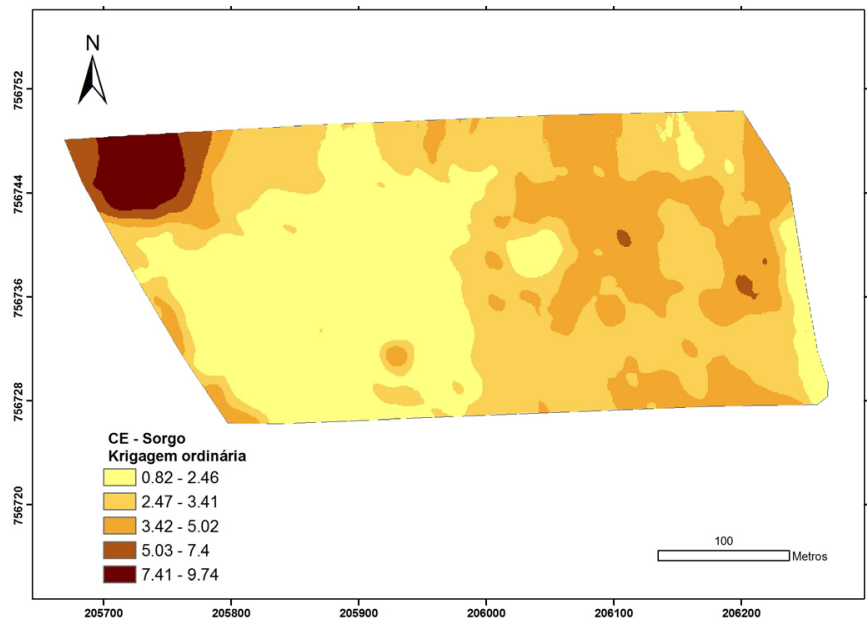

Figura 2. Mapa krigado da condutividade elétrica aparente do solo $\left(\mathrm{mS} \mathrm{m}^{-1}\right)$ de uma área cultivada com sorgo 

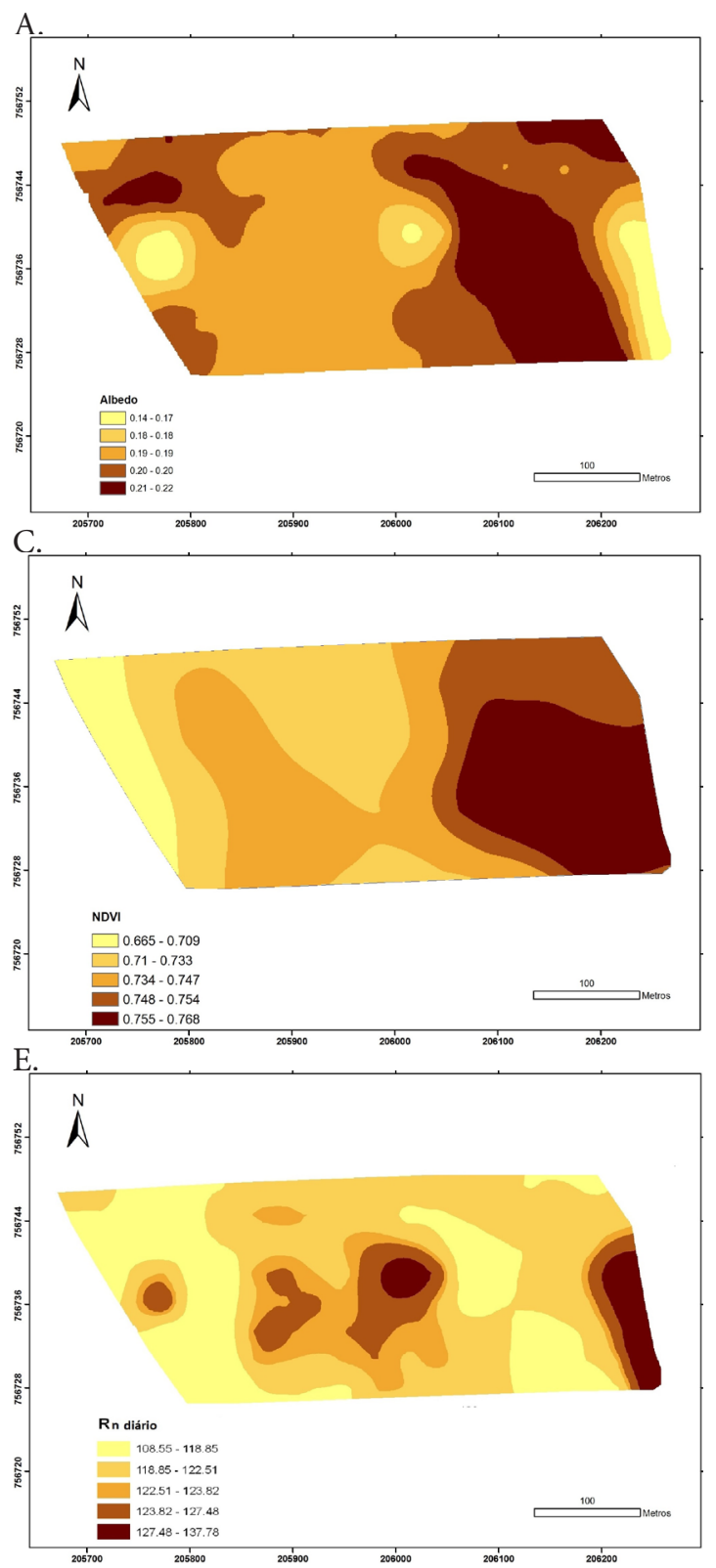

Figura 3. Albedo (A), temperatura da superfície $\left(T s, e m{ }^{\circ} \mathrm{C}\right)$ de radiação diário $\left(\mathrm{Rn}_{\mathrm{d}}\right.$ em W m$\left.{ }^{-2}\right)(\mathrm{E})$ e evapotranspiraça pode observar que os maiores valores de NDVI e IAF estão situados na parte direita da área; o NDVI depende do contraste espectral entre as bandas vermelho e infravermelho próximas e é sensível ao conteúdo de clorofila das folhas e IAF da vegetação (Numata et al., 2007), o que sugere aumentos do NDVI indicando maior produção (áreas mais escuras) da parte aérea.

Para a cultura do sorgo foram observados, na data avaliada, valores médios de IAF da ordem de $4,05 \mathrm{~m}^{2} \mathrm{~m}^{-2}$. Spohr et al. (2005) obtiveram, para o estádio de florescimento do sorgo, IAF variando de 4,2 a 5,8. Peiter \& Carlesso (1996) analisaram o comportamento do sorgo em função de diferentes frações de água disponível no solo e encontraram IAF médio de $4,5 \mathrm{~m}^{2} \mathrm{~m}^{-2}$ após 40 dias da emergência.

$\mathrm{O} \mathrm{Rn}_{\text {diário }}\left(\right.$ Figura $3 \mathrm{E}$ ) variou de 108,55 a 137,78 $\mathrm{W} \mathrm{m}^{-2}$, com média de $120,81 \mathrm{~W} \mathrm{~m}^{-2}$. Este parâmetro foi máximo em uma região central e na região próxima da floresta (delimitação à
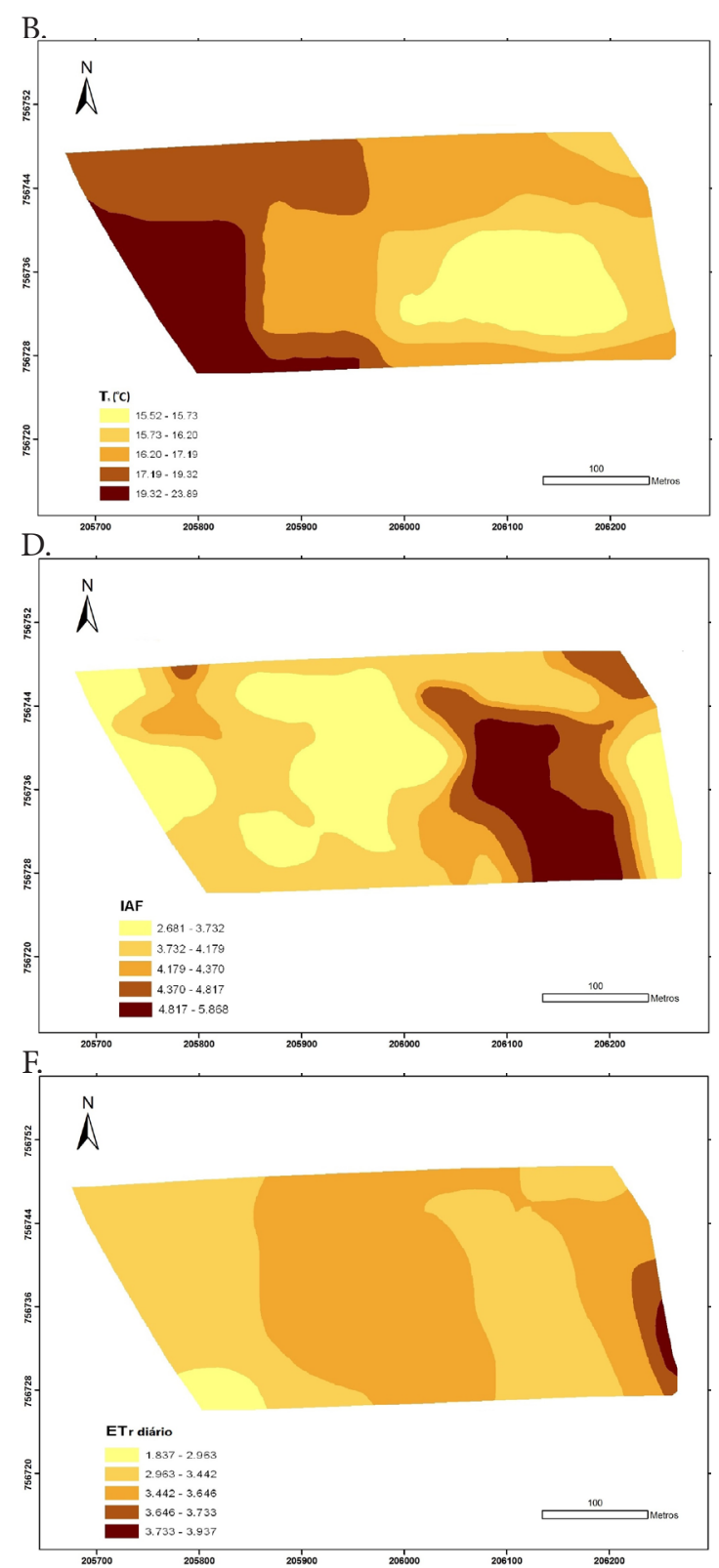

(B), NDVI (C), índice de área foliar (IAF, em m² $\mathrm{m}^{-2}$ (D), saldo ação real diária $\left(E \operatorname{Er}_{\text {dírío }} \mathrm{em} \mathrm{mm} \mathrm{d}^{-1}\right)(\mathrm{F})$ da cultura do sorgo

direita) enquanto a $\mathrm{ETr}_{\text {diário }}$ (Figura $3 \mathrm{~F}$ ) apresentou valor médio de $3,43 \mathrm{~mm} \mathrm{~d}^{-1}$, sendo o valor mínimo e o máximo de $1,84 \mathrm{~mm}$ $\mathrm{d}^{-1}$ (delimitação esquerda inferior) e $3,94 \mathrm{~mm} \mathrm{~d}^{-1}$ (delimitação direita inferior) respectivamente.

Em seu estudo, Steduto \& Albrizio (2005) obtiveram, para a cultura do sorgo, valores de evapotranspiração entre 7,0 $\mathrm{mm} \mathrm{d}^{-1}$ e 7,5 $\mathrm{mm} \mathrm{d}^{-1}$ na antese. No EUA Howell et al. (1997) analisaram vários métodos de estimativa de evapotranspiração em cultura de sorgo no período de 1988 e 1993 e encontraram valores médios de evapotranspiração entre $4,9 \mathrm{~mm} \mathrm{~d}^{-1}$ e 7,93 $\mathrm{mm} \mathrm{d}^{-1}$. Para estudo realizado no Brasil, Assis \& Verona (1991) encontraram, para a cultura do sorgo, valores de evapotranspiração variando de 2,0 $\mathrm{mm} \mathrm{d}^{-1}$ a $7,0 \mathrm{~mm} \mathrm{~d}^{-1}$ de acordo com o método de estimativa e o estádio de desenvolvimento da cultura.

Ao analisar os mapas de $\mathrm{Rn}_{\text {diário }}$ e $\mathrm{ETr}_{\text {diário }}$ não se observou tendência de relação espacial com os parâmetros de solo 
apresentados nas Figuras 1 e 2 porém se ressalta que a densidade de amostragem de solo pode ter sido insuficiente para detectar alguma tendência de relação espacial entre os parâmetros analisados; sugere-se, então, a realização de estudos complementares com grade de pontos mais adensada de forma a elucidar esta questão.

\section{Conclusões}

1. Ocorreu dependência espacial de grau moderada a forte para os dados de CE e biofísicos da superfície da imagem de satélite com alcances variando de 74,4 a 181,1 m.

2. A densidade de amostragem adotada foi insuficiente para uma adequada caracterização da variabilidade espacial de parâmetros do solo.

3. O sensoriamento remoto orbital foi útil para o mapeamento da variabilidade espacial da cultura do sorgo e tem grande potencial para aplicação da Agricultura de Precisão.

\section{Literatura Citada}

Allen, R.; Tasumi, M.; Trezza, R. SEBAL - Surface Energy Balance Algorithms for Land. Advanced Training and Users Manual - Idaho Implementation, version 1.0, 2002. 98p.

Andrade, R. G.; Sediyama, G. C.; Paz, A. R.; Lima, E. P.; Facco, A. G. Geotecnologias aplicadas à avaliação de parâmetros biofísicos do Pantanal. Pesquisa Agropecuária Brasileira, v.47, p.1227-1234, 2012.

Assis, F. N.; Verona, L. A. F. Consumo de água e coeficiente de cultura do sorgo. Pesquisa Agropecuária Brasileira, v.26, p.665-670, 1991.

Ayenew, T. Evapotranspiration estimation using thematic mapper spectral satellite data in the Ethiopian rift and adjacent highland. Journal of Hydrology, v.279, p.83-93, 2003.

Bramley, R. G. V. Lessons from nearly 20 years of precision Agriculture research, development, and adoption as a guide to its appropriate application. Crop \& Pasture Science, v.60, p.197-217, 2009.

Brevik, E.; Fenton, T.; Lazari, A. Soil electrical conductivity as a function of soil water content and implications for soil mapping. Precision Agriculture, v.7, p.393-404, 2006.

Cambardella, C. A.; Moorman, T. B.; Novak, J. M.; Parkim, T. B.; Karlen, D. I.; Turco, R. F.; Koopka, A. E. Field-scale variability of soil properties in Central Iowa Soils. Soil Science Society of America Journal, v.58, p.1501-1511, 1994.

Freeman, K. W.; Girma, K.; Arnall, D. B.; Mullen, R. W.; Martin, K. L.; Teal, R. K.; Raun, W. R. By-plant prediction of corn forage biomass and nitrogen uptake at various growth stages using remote sensing and plant height. Agronomy Journal, v.99, p.530-536, 2007.

Gomes, F. P.; Garcia, C. H. A estatística moderna na pesquisa agropecuária. Piracicaba: Associação Brasileira para Pesquisa da Potassa e do Fosfato, 1984. 160p.

Grego, C. R.; Vieira, S. R. Variabilidade espacial de propriedades físicas do solo em uma parcela experimental. Revista Brasileira de Ciência do Solo, v.29, p.169-177, 2005.
Hatfield, J. L.; Gitelson, A. A.; Schepers, J. S.; Walthall, C. L. Application of spectral remote sensing for agronomic decisions. Agronomy Journal, v.100, p.117-131, 2008.

Howell, T. A.; Steiner, J. L.; Schneider, A. D.; Evett, S. R.; Tolk, J. A. Seasonal and maximum daily evapotranspiration of irrigated winter wheat, sorghum, and corn - southern high plains. Transactions of the ASAE - American Society of Agricultural Engineers, v.40, p.623-634, 1997.

Huete, A. R. A. Soil-adjusted vegetation index (SAVI). Remote Sensing of Enviroment, v.25, p.295-309, 1988.

Johnson, C.; Eskridge, K.; Corwin, D. Apparent soil electrical conductivity: applications for designing and evaluating field-scale experiments. Computers and Electronics in Agriculture, v.46, p.181-202, 2005.

Kilic, K.; Kilic, S.; Kocyigit, R. Assessment of spatial variability of soil properties in areas under different land use. Bulgarian Journal of Agricultural Science, v.18, p.722-732, 2012.

Kravchenko, A. N. Influence of spatial structure on accuracy of interpolation methods. Soil Science Society of America Journal, v.67, p.1564-1571, 2003.

Machado, P. L. O. A.; Bernardi, A. C. C.; Valencia, L. I. O.; Molin, J. P.; Gimenez, L. M.; Silva, C. A.; Andrade, A. G. A.; Madari, B. E.; Meirelles, M. S. P. M. Mapeamento da condutividade elétrica e relação com a argila de Latossolo sob plantio direto. Pesquisa Agropecuária Brasileira, v.41, p.1023-1031, 2006.

Machado, P. L. O. A.; Silva, C. A.; Bernardi, A. C. C.; Carmo, C. A. F. S.; Valencia, L. I. O.; Meirelles, M. S.; Molin, J. P.; Pauletti, V.; Gimenez, L. M. Variabilidade de atributos de fertilidade e espacialização da recomendação de adubação e calagem para a soja em plantio direto. In: Machado, P. L. O. A., Silva, C. A. Bernardi, A. C. C. (ed.). Agricultura de precisão para o manejo da fertilidade do solo em sistema plantio direto. Rio de Janeiro: Embrapa Solos, 2004. p.113-127.

Mota, F. S.; Agendes, M. O. O.; Souza, L. K. Estimativa da evapotranspiração de referência nos principais tipos de climas do Brasil com instrumentos de baixo custo. Revista Brasileira de Meteorologia, v.6, p.479-486, 1991.

Numata, I.; Roberts, D. A.; Chadwick, O. A.; Schimel, J.; Sampaio, F. R.; Leonidas, F. C.; Soares, J. V. Characterization of pasture biophysical properties and the impact of grazing intensity using remotely sensed data. Remote Sensing of Environment, v.109, p.314-327, 2007.

Peiter, M. X.; Carlesso, R. Comportamento do sorgo granífero em função de diferentes frações da água disponível no solo. Ciência Rural, v.26, p.51-55, 1996.

Primavesi, A. C.; Andrade, A. G.; Alves, B. J. R.; Rosso, C.; Batista, E. M.; Prates, H. T.; Ortiz, F. R.; Mello, J.; Ferraz, M. R.; Linhares, N. W.; Machado, P. L. O. A.; Moeller, R.; Alves, R. C. S.; Silva, W. M. Métodos de análise de solo. In: Nogueira, A. R. A.; Souza, G. B. Manual de laboratórios: Solo, água, nutrição vegetal, nutrição animal e alimentos. São Carlos: Embrapa Pecuária Sudeste, 2005. p.67-130.

Raij, B. van.; Cantarella, H.; Quaggio, J. A.; Furlani, A. M. C. (ed.). Recomendações de adubação e calagem para o Estado de São Paulo. 2.ed. Campinas: IAC, 1996. 285p. Boletim Técnico, 100 
Rouse, J. W.; Haas, R. H.; Schell, J. A.; Deering, D. W. Monitoring vegetation systems in the great plains with ERTS. In: Earth Resources Technology Satellite -1 Symposium, 3, 1973, Washington. Proceedings... Washington: NASA, Goddard Space Flight Center 1, 1973. p.309-317.

Spohr, R. B.; Maggi, M. F.; Carlesso, R. Kunz, J. H.; Fiorin, T. Irrigação na produção de silagem de milho e sorgo, em sucessão ao arroz de terras altas. Revista Brasileira de Engenharia Agrícola e Ambiental, v.9, p.469-474, 2005.

Steduto, P.; Albrizio, R. Resource use efficiency of field-grown sunflower sorghum, wheat and chickpea II. Water use efficiency and comparison with radiation use efficiency. Agricultural and Forest Meteorology, v.130, p.269-281, 2005.

Trangmar, B. B.; Yost, R. S.; Uehara, G. Application of geostatistics to spatial studies of soil properties. Advances in Agronomy, v.38, p.45-94, 1985.
Vicente, L. E.; Gomes, D.; Victoria, D. C.; Garçon, E. A. M.; Bolfe, E. L.; Andrade, R. G.; Silva, G. B. S. Séries temporais de NDVI do sensor SPOT Vegetation e algoritmo SAM aplicados ao mapeamento de cana-de-açúcar. Pesquisa Agropecuária Brasileira, v.47, p.1337-1345, 2012.

Vieira, S. R. Geoestatística em estudos de variabilidade espacial do solo. In: Novais, R. F.; Alvares, V. V. H.; Schaeffer, C. E. G. R. (eds.) Tópicos em ciência do solo. Viçosa: Sociedade Brasileira de Ciência do Solo, 2000. p.1-54.

Vieira, S. R.; Millete, J.; Topp, G. C.; Reynolds, W. D. Handbook for geostatistical analysis of variability in soil and climate data. In: Alvares, V. V. H.; Schaeffer, C. E. G. R.; Barros, N. F.; Mello, J. W. V.; Costa, L. M. (ed). Tópicos em ciência do solo. Viçosa: Sociedade Brasileira de Ciência do Solo, v.2, 2002. p.1-45. 